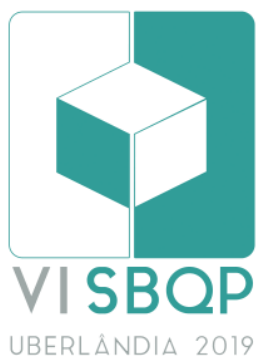

\title{
AVALIANDO ENGAJAMENTO EM HABITAÇÃO DE INTERESSE SOCIAL: UMA BUSCA POR RESILIÊNCIA
}

\author{
VASCONCELLOS, Paula Barcelos \\ Universidade Federal de Uberlândia, e-mail: paulab_vas@yahoo.com.br \\ VILLA, Simone Barbosa \\ Universidade Federal de Uberlândia, e-mail: simonevilla@yahoo.com
}

\begin{abstract}
RESUMO
Perante o estado de vulnerabilidade atual, principalmente no que tange a Habitação de Interesse Social, é necessário considerar a resiliência como uma capacidade adaptativa que o combate, reduzindo/sanando os pequenos e constantes impactos que essas comunidades sofrem, os quais são prejudiciais a longo prazo. Neste cenário, o presente trabalho parte de uma pesquisa de mestrado, intitulada "CO-PRODUZINDO RESILIÊNCIA EM HABITAÇÃO DE INTERESSE SOCIAL: Como avaliar a resiliência através do engajamento?", a qual se estrutura através do conceito de resiliência social e coprodução, bem como seus impactos no ambiente construído, tendo como estudo de caso um Bairro de Habitação Social em Uberlândia (MG). A dissertação tem como objetivo, a partir de pesquisa teórica e aplicação de Avaliação Pós-Ocupação e Coproduções, investigar os indicadores de resiliência do bairro a partir da ótica do Engajamento, identificando quais impactos e qualidades determinam essa resiliência. Este artigo apresenta parte da pesquisa com foco no processo de construção da ferramenta de Avaliação Pós-Ocupação. Com os resultados, pretende-se fomentar a discussão sobre os indicadores de resiliência social no ambiente construído, bem como sobre as ferramentas de avaliação.
\end{abstract}

Palavras-chave: Resiliência, Avaliação Pós-Ocupação, Habitação Social, Coprodução, Engajamento.

\begin{abstract}
Given the present state of vulnerability, especially in relation to Social Housing, it is necessary to consider resilience as an adaptive capacity to combat it, reducing/healing the small and constant impacts that these communities suffer, which are harmful in the long run. In this scenario, the present work originates from a master's research, entitled "CO-PRODUCING RESILIENCE IN SOCIAL HOUSING: How to evaluate resilience through engagement?", which is structured through the concept of social resilience and coproduction, and its impacts on the built environment, having as a case study a Social Housing Neighborhood in Uberlândia (MG). The dissertation aims, with theoretical research, application of Post-Occupancy Evaluation and Coproduction tools, to investigate the neighborhood's resilience Engagement indicators, identifying which impacts and qualities determine this resilience. This article presents part of the research focused on the process of construction of the Post-Occupancy Evaluation tool. With the results we intend to encourage a discussion about social resilience indicators in the built environment, as well as on the evaluation tools.
\end{abstract}

Keywords: Resilience, Post-Ocupanccy Evaluation, Social Housing, Co-production, Engagement.

VASCONCELLOS, P. B.; VILLA, S. B. Avaliando engajamento em Habitação de Interesse Social: uma busca por resiliência. In: SIMPÓSIO BRASILEIRO DE QUALIDADE DO PROJETO NO AMBIENTE CONSTRUÍDO, 6., 2019, Uberlândia. Anais... Uberlândia: PPGAU/FAUeD/UFU, 2019. p. 1153-1165. DOI https://doi.org/10.14393/sbqp19105. 


\section{INTRODUÇÃO}

O presente trabalho apresenta resultados parciais de pesquisa de mestrado em andamento, intitulada "CO-PRODUZINDO RESILIÊNCIA EM HABITAÇÃO DE INTERESSE SOCIAL: Como avaliar a resiliência através do engajamento?". Pesquisa esta, que deriva de uma pesquisa maior, entitulada "[RES_APO] Método de Análise da Resiliência e Adaptabilidade em Conjuntos Habitacionais Sociais através da Avaliação Pós-Ocupação e Coprodução"l, desenvolvida pelos grupos [MORA] pesquisa em habitação da FAUeD/UFU e [Design Engagement and Practice] da SSOA da Universidade de Sheffield TUoS. O objetivo da presente pesquisa de mestrado consiste em identificar indicadores de resiliência em Habitação de Interesse Social (HIS) a partir da ótica do Engajamento da comunidade em questão, identificando os impactos e as qualidades que determinam tal resiliência, na sua relação com seus moradores dentro do ambiente construído, através de pesquisa avaliativa e análise de práticas colaborativas. Para tanto, tem como estudo de caso o Residencial Sucesso Brasil, parte do conjunto habitacional de interesse social (CHIS) Shopping Park, na cidade de Uberlândia, MG.

Perante diversos impactos e mudanças, a baixa qualidade da produção de Habitação de Interesse Social (HIS) ainda permanece como um dos principais fatores do cenário de vulnerabilidade social que presenciamos (STEVENSON, PETRESCU, 2016; VILLA et al, 2017). Frente a esses conjuntos marginalizados, a pesquisa busca, a partir do conceito de resiliência, identificar como estas comunidades se adaptam a esses diversos impactos e mudanças, bem como estas relações acontecem no ambiente construído. Para tanto, o artigo em questão apresenta parte deste estudo, com foco nas ferramentas de avaliação construídas a partir do atributo de investigação - Engajamento. Desta forma são apresentados: (i) fundamentação teórica; (ii) metodologia; (iii) resultados parciais; (iv) considerações finais.

\section{FUNDAMENTAÇÃO}

\subsection{HIS no Brasil: Um cenário problema}

A falta de qualidade do que se é ofertado, bem como a ineficiência das políticas públicas fazem com que o déficit habitacional ainda seja um problema que perdure em território nacional (VILLA, 2010; VILLA; VASCONCELLOS, 2015). De acordo com a Fundação Getúlio Vargas, o PMCMV entregou, dentro do período de 2009 a 2019, cerca de 4,4 milhões de unidades. Entretanto, após dez anos de lançamento do programa, este número continua insuficiente. O déficit habitacional continuou a crescer, passando de 6 milhões para 7,7 milhões em 2018 (BOHM, 2018). Além dos números, o problema se torna mais alarmante quando se leva em consideração a qualidade das habitações já existentes, caracterizadas pela baixa qualidade construtiva e espacial, a qual negligencia as transformações dos perfis familiares e suas reais necessidades; além da periferização dos conjuntos habitacionais e falta de infraestrutura (TRAMONTANO, 1993; VILLA, 2010).

\footnotetext{
1 VILLA, S. B.; et al. [RES_APO] Método de análise da resiliência e adaptabilidade em conjuntos habitacionais sociais através da avaliação pós-ocupação e coprodução. RELATÓRIO FINAL DE PESQUISA: Uberlândia: Universidade Federal de Uberlândia; Universidade de Sheffield, 2017.
} 
Entendendo este cenário, é preciso buscar novas óticas e aprimorar ferramentas avaliativas de modo a buscar de fato, mudanças positivas no cenário habitacional nacional.

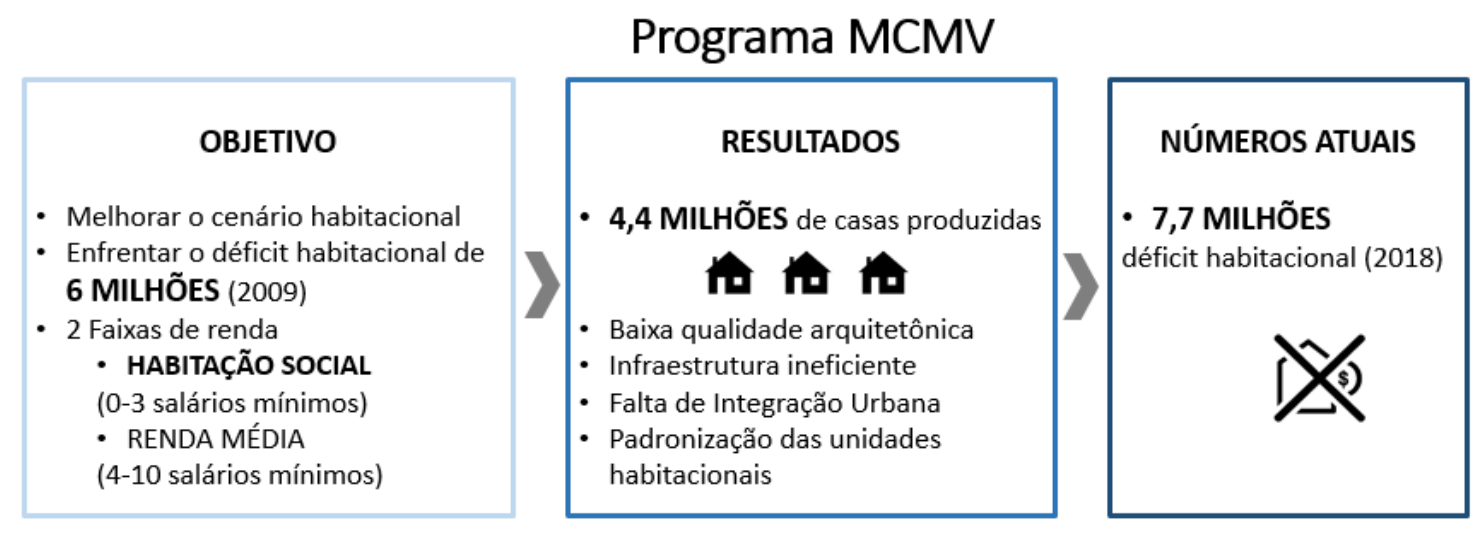

Figura 1 - Resultados do Programa Minha Casa Minha Vida Fonte: Autoras (2019)

\subsection{Resiliência: Uma resposta à vulnerabilidade}

O termo Resiliência se popularizou nos últimos anos, principalmente devido a sua utilização das novas agendas urbanas (MEEROW; NEWELL; STULTS, 2015). O termo é considerado como um boundary object ("objeto de limite" em tradução literal), pois atua de forma intercambiável entre diversas áreas do conhecimento e suas interfaces (MEEROW; NEWELL, 2016).

\section{Evolução do conceito de Resiliência}

\begin{tabular}{|c|c|c|}
\hline & $\begin{array}{l}\text { - Conotação primária - propriedade que } \\
\text { faz com que um objeto retorne ao seu } \\
\text { estado original após ser distorcido por } \\
\text { uma força.(Valle \& Garcia, 2015) }\end{array}$ & \multirow{3}{*}{$\begin{array}{l}\text { Conceito tradicional de } \\
\text { resiliência geralmente } \\
\text { associado à capacidade de } \\
\text { um sistema absorver } \\
\text { perturbações e reorganizar- } \\
\text { se quando sujeito a } \\
\text { mudanças, mantendo ao } \\
\text { mesmo tempo suas funções } \\
\text { essenciais, estrutura, } \\
\text { identidade e mecanismos. } \\
\text { (Walker et al., 2004; Thackara, 2008) }\end{array}$} \\
\hline \multirow{2}{*}{\multicolumn{2}{|c|}{$\begin{array}{l}\text { - Década de } 1970 \text { (perspectiva mais } \\
\text { ecológica) - capacidade de um sistema } \\
\text { de absorver, e até de se beneficiar, de } \\
\text { impactos incidentais, sem que o } \\
\text { sistema sofra danos permanentes em } \\
\text { sua estrutura ou funcionamento. } \\
\text { (Holling, 1973) }\end{array}$}} & \\
\hline & & \\
\hline - & \multicolumn{2}{|c|}{$\begin{array}{l}\text {-Esta pesquisa considera a Resiliência como a capacidade de } \\
\text { recuperação adaptativa frente a impactos diversificados, de ordem } \\
\text { física, ambiental ou social.. } \\
\text { - Identifica os recursos e a adaptabilidade que uma comunidade pode } \\
\text { usar para superar esses impactos, aproveitando seus recursos } \\
\text { inerentes.(Maguire \& Cartwright, 2008; Adger, 2000) }\end{array}$} \\
\hline
\end{tabular}

Figura 2 - Evolução do conceito de Resiliência Fonte: Autoras (2019) 
A abordagem utilizada nesta pesquisa tem conotação positiva, compreendendo como resiliência a capacidade de adaptação ou de recuperação de diferentes impactos (naturais, sociais, físicos). Tal conceito apresenta uma abordagem mais dinâmica, considerando como uma comunidade pode utilizar recursos e se adaptar, frente aos problemas que podem resultar da mudança (MAGUIRE, CARTWRIGHT, 2008). Desta forma, a presente pesquisa se ancora no conceito de Resiliência Social, na qual a forma de abordagem, ao invés de se firmar em fatores externos para solucionar as vulnerabilidades, se estrutura nas capacidades inerentes de uma comunidade em lidar com problemas/alterações e constantemente se adaptar (ADGER, 2000).

\subsection{APO: Avaliando a Resiliência através do Engajamento}

A Avaliação Pós-Ocupação é uma ferramenta já consolidada, no que se diz respeito à qualidade do projeto, tanto em cenário nacional como internacional (ONO et al., 2018; LEAMAN; STEVENSON, BORDASS, 2010; MALLORY-HILL; PREISER; WATSON 2012). Se mostra importante também na função de revelar as relações entre o comportamento humano e sua relação com o espaço construído, estabelecendo índices de satisfação e desempenho que contribuem para a melhoria dos projetos (VILLA; SARAMAGO; GARCIA, 2015).

A avaliação da presente pesquisa - associada a metodologias de prática colaborativa - se estrutura a partir do conceito de Engajamento, considerando-o como necessário dentro do conceito de resiliência social para compreender a força das relações e como isso afeta o ambiente construído no cenário atual. Para tanto, considera como engajamento a participação ativa em assuntos e circunstâncias, tendo impacto direto e demonstrável na produtividade e performance que se traduz em resultados - principalmente nas coproduções. Bem como a força dos relacionamentos de um indivíduo e da maneira como eles funcionam dentro de sua comunidade e do ambiente construído (SCHAUFELI; BAKKER, 2003; ANDERSON et al., 2016).

Como visto anteriormente, os conjuntos de HIS são geralmente localizados em regiões periféricas, marcados pela segregação espacial e social resultante de uma prática econômica guiada pelo lucro (MARICATO, 2000; VILLA; OLIVEIRA; SARAMAGO, 2013). Desta forma, se faz necessário a avaliação para compreender como se dão as relações destas "comunidades forçadas", de modo a descobrir formas de atuação dentro da comunidade que aumentem seu nível de engajamento e, consequentemente, de resiliência, impactando positivamente o ambiente construído.

\section{METODOLOGIA}

\subsection{Metodologia de atuação}

A metodologia se estrutura no Design Science Research (DRESCH, LACERDA, ANTUNES JR., 2015), metodologia de pesquisa robusta que se estrutura nas etapas de (i) Conscientização - levantamento do problema (ii) Análise Sistemática da literatura (iii) Identificação da Classe de Problemas (iv) Construção do artefato - ferramenta de avaliação, no caso desta pesquisa. A pesquisa possui abordagem qualitativa, composta por pesquisa avaliativa 
(APO) e análise de práticas colaborativas (coproduções) e sua relação/impacto no ambiente construído.

Quadro 1 - Metodologia e Referências

\begin{tabular}{|c|c|c|c|}
\hline & Atividade & Objetivo & Referências \\
\hline \multirow{4}{*}{ 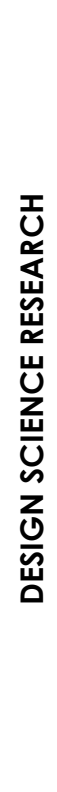 } & $\begin{array}{c}\text { Revisão e Pesquisa } \\
\text { Bibliográfica, e } \\
\text { Fundamentação Teórica }\end{array}$ & $\begin{array}{c}\text { Conceituação de resiliência e } \\
\text { coprodução para acessar } \\
\text { objetivos principais da pesquisa }\end{array}$ & $\begin{array}{l}\text { MAGUIRE; CARTWRIGHT; } \\
\text { 2008; GARCIA; VALE, } 2017\end{array}$ \\
\hline & $\begin{array}{c}\text { Análise dos dados de } \\
\text { pesquisas já desenvolvidas }\end{array}$ & $\begin{array}{c}\text { Analisar experiências anteriores } \\
\text { para auxiliar na formação dos } \\
\text { indicadores e formas de atuação } \\
\text { na comunidade }\end{array}$ & $\begin{array}{l}\text { VILLA et al., 2017; } \\
\text { PETRESCU; PETCOU; } \\
\text { BAIBARAC, } 2016\end{array}$ \\
\hline & $\begin{array}{c}\text { Avaliação Pós-Ocupação } \\
\text { (APO) }\end{array}$ & $\begin{array}{c}\text { Identificar impactos no ambiente } \\
\text { construído, bem como a visão dos } \\
\text { usuários; Entrevistas, conversas com } \\
\text { o objetivo de perceber a visão dos } \\
\text { usuários e agentes locais; Registro } \\
\text { por observação das atividades } \\
\text { realizadas }\end{array}$ & $\begin{array}{l}\text { VILLA; ORNSTEIN, 2013; } \\
\text { VILLA; SARAMAGO; } \\
\text { GARCIA, 2015; LOPES et } \\
\text { al., 2002; BEAUD; WEBER, } \\
2007\end{array}$ \\
\hline & Coprodução & $\begin{array}{c}\text { Práticas colaborativas em conjunto } \\
\text { com os moradores, para atuação } \\
\text { dentro da comunidade }\end{array}$ & $\begin{array}{l}\text { PETCOU; PETRESCU, 2015; } \\
\text { STEVENSON; PETRESCU, } \\
\text { 2016; PETCOU; BAIBARAC, } \\
2016\end{array}$ \\
\hline
\end{tabular}

Fonte: Autoras (2019)

Sendo parte de uma pesquisa maior, o presente estudo tem como ponto de partida uma base de dados já levantadas. Busca identificar, a partir da ótica do atributo Engajamento, impactos incidentes na comunidade vulnerabilidades ou capacidades adaptativas - possibilitando a identificação de estratégias de atuação (Recomendações para resiliência), fundamentadas em parâmetros estabelecidos. Para tanto, foram definidos cinco indicadores para o atributo Engajamento:

- Fazer parte - Estar envolvido em seu contexto, encontrando sentido em ali residir, tendo senso de entusiasmo, inspiração e orgulho.

- Compartilhar - Compartilhamento de conhecimento e recursos, de forma a proporcionar aprendizado a nível individual e coletivo, capacitando a família e a comunidade a se adaptar.

- Comunicação - Laços e formas de comunicação tanto entre membros de uma mesma residência, bem como com outros moradores e agentes externos do bairro.

- Motivação - Forças que induzem os moradores a agir de determinada maneira, de modo a assegurar o cumprimento de uma necessidade específica.

- Segurança - Segurança é o estado de ser "seguro", a condição de ser protegido contra danos ou outros resultados indesejáveis.

\subsection{Estudo de caso}

A área de estudo é caracterizada por ser o maior empreendimento de habitação social da cidade de Uberlândia, MG. Localizado na região sul, o 
bairro Shopping Park abriga 3000 unidades habitacionais térreas do PMCM, dentro da faixa de renda 1, construídas nos anos de 2010-2013. O empreendimento é composto por 12 residenciais, sendo o Residencial Sucesso Brasil, a área de estudo da pesquisa (Figura 1). Apesar da grande iniciativa, 0 local apresenta diversos problemas sociais, construtivos e de infraestrutura que impedem os moradores de conseguirem uma qualidade de vida adequada.

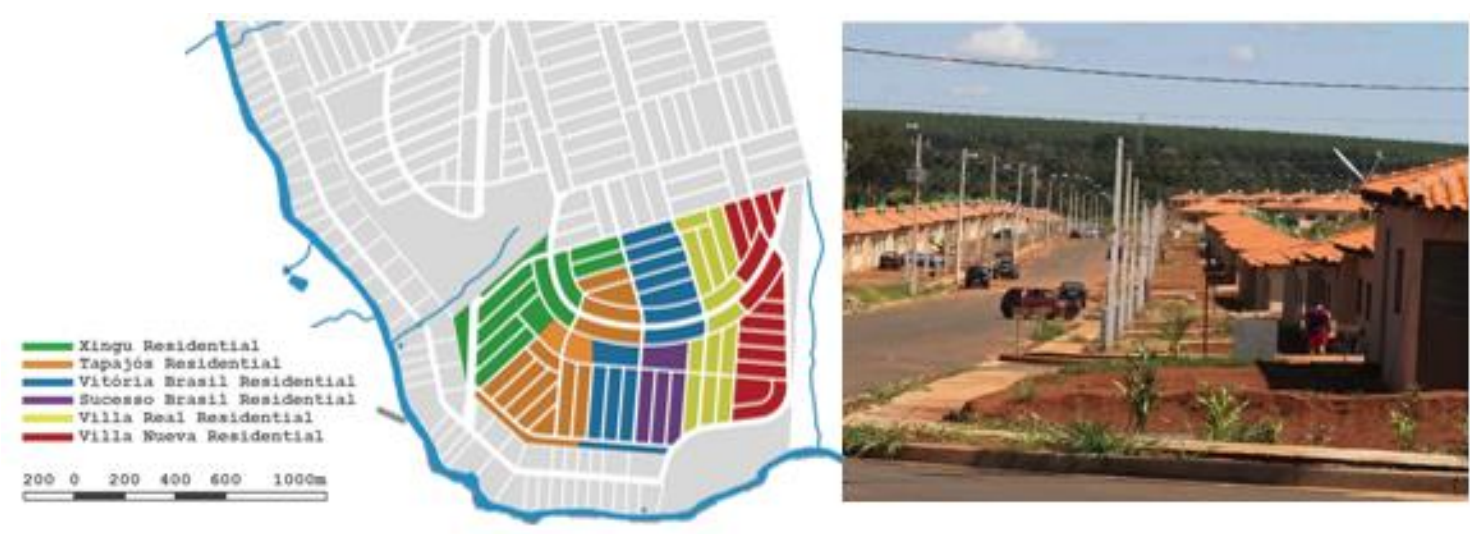

Figura 3 - Área de estudo - Bairro Shopping ParK Fonte: Villa et at. (2017), adaptado pelas autoras (2019)

\section{RESULTADOS PARCIAIS}

\subsection{Identificando as Vulnerabilidades e Capacidades Adaptativas}

\begin{tabular}{|c|c|c|}
\hline \multicolumn{3}{|c|}{ CENÁRIOS } \\
\hline CENÁRIO 1 (2013) & CENÁRIO 2 (2016/2017) & CENÁRIO 3 (2018/2019) \\
\hline \multicolumn{3}{|c|}{ ATIVIDADES REALIZADAS } \\
\hline \multirow[t]{4}{*}{$\begin{array}{l}\text { Coleta geral de } \\
\text { dados }\end{array}$} & Coleta geral de dados & Coproduções \\
\hline & $\begin{array}{c}\text { APOs (questionários + } \\
\text { walkthroughs) }\end{array}$ & $\begin{array}{c}\text { Ferramenta de } \\
\text { Avaliação }\end{array}$ \\
\hline & Coproduções & \\
\hline & $\begin{array}{l}\text { - "Venha tomar café com } \\
\text { - "Q gente" } \\
\text { "Queremos saber mais } \\
\text { - "Vobre você e seu bairro" } \\
\text { - " } \quad \text { "Pairro. Paño participe!" ações no } \\
\text { ações" }\end{array}$ & $\begin{array}{l}\text { - "Workshop de plantio } \\
\text { - "We mudas" } \\
\text { "Workshop de móvel } \\
\text { de palete" }\end{array}$ \\
\hline
\end{tabular}

Figura 4 - Cenários e atividades realizadas -

Fonte: Autoras (2019)

Dentro do contexto de Resiliência, a vulnerabilidade em unidades habitacionais de interesse social refere-se ao seu estado de 
sensibilidade/susceptibilidade à determinada(s) ameaça(s), derivando, principalmente, de características inerentes ao projeto entregue e da situação da edificação no momento da incidência dessa(s) ameaça(s), que comprometem sua capacidade de resistir, adaptar-se e transformar-se. Da mesma forma, a Capacidade Adaptativa refere-se aos recursos e a adaptabilidade que uma comunidade usa para superar esses impactos.

Deste modo, foram realizados o levantamento de dados e avalições do estudo de caso a partir de 3 diferentes cenários (Figura 4): (i) Cenário 1 (2013) Quando a casa foi entregue (2013); (ii) Cenário 2 (5 anos depois) Aplicação de questionários e início da Coprodução; (iii) Cenário 3 (2018/2019) - Aplicação de um novo questionário, após a realização das Coproduções.

A coleta de dados e aplicação de questionários/walkthroughs derivou de uma pesquisa maior - [RES_APO 2 e 3 $]^{2}$, buscando identificar as condições do cenário atual no bairro, as ameaças incidentes sobre as casas e os moradores, e também as capacidades adaptativas desenvolvidas por essa comunidade, advindas desses impactos.

Quadro 2 - Descrição das ferramentas de APO

\begin{tabular}{|c|c|}
\hline \multicolumn{2}{|r|}{ FERRAMENTAS APO } \\
\hline \multicolumn{2}{|r|}{ Questionário } \\
\hline $\begin{array}{c}\text { Descrição e } \\
\text { objetivos }\end{array}$ & $\begin{array}{l}\text { Método quantitativo que permite a coleta de dados por meio de } \\
\text { perguntas respondidas pelos moradores do bairro Shopping Park. } \\
\text { Esse questionário aborda perguntas relativas às ordens de avaliação da } \\
\text { pesquisa: ordem socioeconômica, ordem natural-climática, ordem } \\
\text { físico-arquitetônica e ordem físico-urbanística. Portanto, o questionário } \\
\text { com os moradores permite a coleta de informações relativas a dados } \\
\text { demográficos, renda, escolaridade, comunicação, saúde, violência, } \\
\text { consumo de água/energia, produção de alimentos, lixo/polvição, } \\
\text { características construtivas da habitação, qualidade urbanística do } \\
\text { entorno, entre outros fatores, envolvendo a percepção dos moradores } \\
\text { participantes. }\end{array}$ \\
\hline Amostragem & 40 casas \\
\hline Data/Local & 27/03/2018 a 20/04/2018 - Residencial Sucesso Brasil \\
\hline \multicolumn{2}{|r|}{ Walkthrough } \\
\hline $\begin{array}{c}\text { Descrição e } \\
\text { objetivos }\end{array}$ & $\begin{array}{l}\text { É um método tanto qualitativo como quantitativo para a medição, } \\
\text { análise e identificação de aspectos positivos e negativos do ambiente } \\
\text { construído, além de sua situação atual, avaliando os eixos temáticos: i) } \\
\text { lote e ii) unidade habitacional. }\end{array}$ \\
\hline Amostragem & 7 casas \\
\hline Data/Local & 25/04/2018 a 16/05/2018 - Residencial Sucesso Brasil \\
\hline
\end{tabular}

Fonte: VILLA et al. (2017), adaptado pelas autoras (2019)

As Coproduções tiveram como objetivo maior identificar as principais vulnerabilidades e potencialidades do local segundo a visão dos próprios residentes, bem como proporcionar a aproximação entre o grupo de pesquisa e a comunidade local. Para tanto foram realizadas reuniões tanto no centro comunitário, quanto nas residências dos próprios moradores, realizando

2 VILLA, S. B. et al. [RES_APO 2 e 3] Resiliência e Adaptabilidade em Conjuntos Habitacionais Sociais Através da Coprodução. RELATÓRIO PARCIAL DE PESQUISA: Uberlândia: Universidade Federal de Uberlândia; 2018. 
dinâmicas e atividades (mapeamento, desenhos, conversas, workshops) que explorassem as reflexões dos moradores sobre o lugar onde moram, potencialidades e problemas do bairro segundo os mesmos. Ao se concentrar nos problemas de moradia e em como poderiam ser resolvidos, os moradores se mostraram mais interessados e participativos, possibilitando a validação das informações levantadas com os instrumentos de APO.

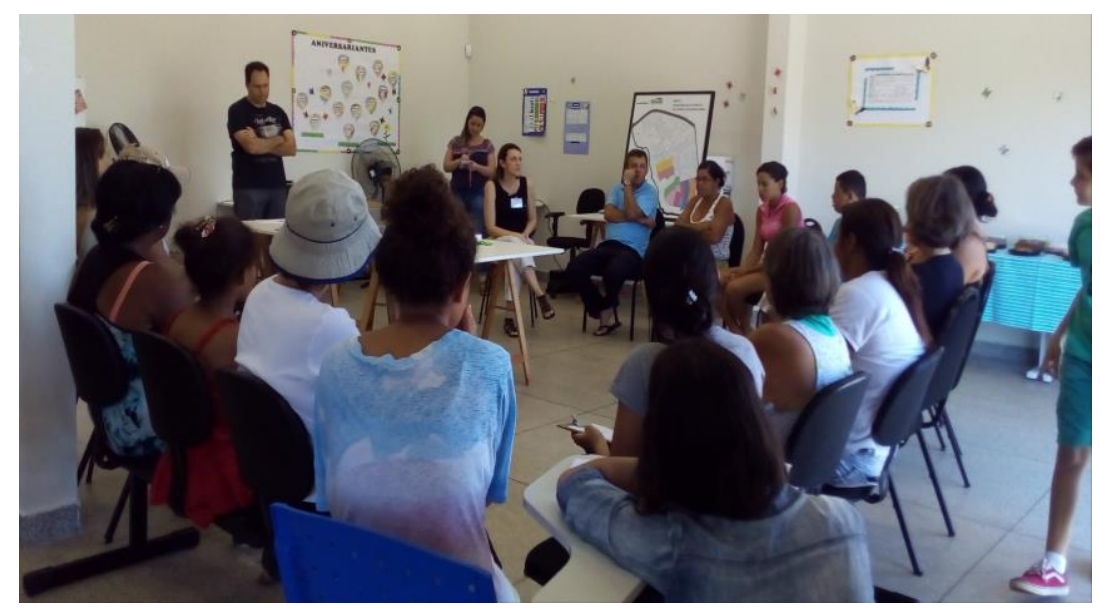

Figura 5 - Realização de uma das coproduções Fonte: VILLA et at. (2017)

A partir do estabelecimento dessa relação mais próxima entre o grupo de pesquisa e a comunidade, foi possível identificar, associado às informações levantadas por meio da APO e da coleta de dados, algumas informações importantes sobre o atributo de Engajamento. Dessa forma, foi feita uma análise dos Impactos (Vulnerabilidades e capacidades adaptativas) a partir de cada indicador do atributo Engajamento, com objetivo de auxiliar na construção da ferramenta final de avaliação, que tem como objetivo avaliar resiliência através do engajamento em uma comunidade.

\section{Quadro 3 - Impactos Identificados}

\begin{tabular}{|l|}
\hline \multicolumn{1}{|c|}{ Impactos por indicador } \\
\hline \multicolumn{1}{|c|}{ FAZER PARTE } \\
\hline A nível de bairro/vizinhança \\
-91,4\% utilizam a casa como espaço de lazer e "refúgio". \\
- 57,5\% sentem falta de áreas de lazer, com 50\% afirmando que isso se deve à falta de serviços \\
e variedades de atividades ofertadas \\
A nível de unidade habitacional \\
-31,6\% sentem falta de privacidade. \\
-Dimensões reduzidas limitam a individualidade e recolhimento \\
A nível familiar \\
-perfil majoritário família nuclear (40\%), seguido da família monoparental (17,5\%) \\
-maioria dos respondentes são mulheres (75\%), sendo 52,5\% chefes de sua família \\
\hline \multicolumn{1}{c|}{ COMPARTILHAR } \\
\hline -Instituições importantes, que atuam neste compartilhamento de conhecimento e recursos: \\
$\begin{array}{l}\text { ONG Estação Vida e a Missão Sal da Terra/ CRAS (Centro de Referência e Assistência Social)/ } \\
\text { CEU (Centro de Artes e Esporte Unificado) } \\
\text {-87,5\% afirmaram ter algum tipo de planta em sua residência - Ação de Plantio de Mudas } \\
\text { permitiu o contato entre moradores e troca de mudas, vasos, e conhecimento }\end{array}$ \\
\hline
\end{tabular}




\section{COMUNICAÇÃO}

$-95 \%$ possui celular, sendo que $85 \%$ o utilizam para falar e $67,5 \%$ o utilizam para acessar a internet

$-35 \%$ dos respondentes possuem tablets/computadores e $42,5 \%$, possuem tv a cabo

-Falta de privacidade acústica prejudica convivência entre vizinhos

-alguns dos respondentes se queixaram da dificuldade $(25 \%)$ ou a falta $(12,5 \%)$ de interação com os vizinhos

-não há uma forte interação dos moradores do residencial com as lideranças da Associação de Moradores do bairro Shopping Park

\section{MOTIVAÇÃO}

-50\% possui renda entre $\mathrm{R} \$ 1000,00$ e $\mathrm{R} \$ 2000,00$ - funciona como um motivador na busca por maior capacitação

coproduções e atividades colaborativas funcionaram como motivação, devido às possibilidades de melhorias apresentadas

-Maior interação com os moradores a partir da aplicação presencial dos questionários, e das coproduções e ação de Plantio de Mudas

-Sensação de pertencimento derivada da realização do "sonho da casa própria" justifica iniciativa dos moradores em adaptar suas residências.

$-67,5 \%$ estão satisfeitos de maneira geral com sua residência e $77,5 \%$ se adaptaram bem a ela

\section{SEGURANÇA}

$-50 \%$ dos entrevistados afirmou se sentir segura no bairro, enquanto $32,5 \%$ se sentem inseguros e $17,5 \%$ muito inseguros

-72,5\% dos respondentes afirmaram que construíram muros na busca por maior segurança, sendo que $100 \%$ das casas analisadas possuíam muros externos

$-80 \%$ não visitam o rio, com $45 \%$ afirmando que é porque o consideram perigoso

-a partir da coprodução, sugestão da instalação de um posto policial no CEU

-roubo domiciliar apresenta os maiores índices dentre os tipos de ações identificadas

-uso e tráfico de drogas são encarados como uma forma de ascenção social e capacitação

\section{REFERÊNCIAS}

-Anderson, Ruggeri, Steemers and Huppert: Lively Social Space, Well-Being Activity, and Urban Design

-A Manager's Guide to Evaluating Citizen Participation - Syracuse University, 2012

-Sabioni, Ferreira, Braga e Almeida: Contextos (in)adequados para o engajamento cidadão no controle social, 2016

-Health and Wellbeing in Homes, Uk Green Building Council, 2016

Fonte: Autoras (2019)

\subsection{A Ferramenta de avaliação}

A partir dos impactos identificados, busca-se o desenvolvimento de uma ferramenta de avaliação, que possa identificar as relações sociais e impactos no ambiente construído a partir dos indicadores do atributo engajamento. A mesma será aplicada através de uma ferramenta digital ${ }^{3}$ - tablet - de modo a tornar o processo de avaliação mais direto, prático e interativo, bem como facilitar a coleta e análise de resultados.

Os resultados obtidos indicarão, dentro do estudo de caso, as categorias (indicadores do atributo Engajamento) mais prejudicadas. A partir destes dados, serão indicadas recomendações e soluções direcionadas ao próprio morador, dentro de uma plataforma digital (blog) de simples manuseio, para

\footnotetext{
${ }^{3}$ Insere-se em pesquisas do grupo [MORA] Pesquisa em Habitação sobre Inovação Tecnológica em APO. Disponível em: <https://morahabitacao.com/pesquisas-em-andamento-2/inovacaotecnologica-tecnologic-innovation/>.
} 
que o mesmo possa realizar as ações recomendadas para amenizar e/ou sanar estes problemas.

Quadro 4 - Objetivos do questionário

\begin{tabular}{|c|c|}
\hline Indicador & Informações a serem analisadas \\
\hline FAZER PARTE & $\begin{array}{l}\text { - Intenção de continuar morando no bairro } \\
\text { - Nível de relacionamento com vizinhança } \\
\text { - Reformas e alterações para melhor adaptação, conforto familiar } \\
\text { - Nível de dificuldade de adaptação de mobiliário }\end{array}$ \\
\hline COMPARTILHAR & $\begin{array}{l}\text { - Atividades de ensino } \\
\text { - Formas de apropriação do espaço para aprendizado e } \\
\text { compartilhamento de conhecimento }\end{array}$ \\
\hline COMUNICAÇÃO & $\begin{array}{l}\text { - Acesso à informação em geral } \\
\text { - } \quad \text { Acesso à informação sobre o que acontece no bairro } \\
\text { - Encontro regulares com amigos e vizinhos } \\
\text { - Apropriação do espaço para melhor relacionamento com vizinhos } \\
\text { - Participação em eventos da comunidade }\end{array}$ \\
\hline MOTIVAÇÃO & 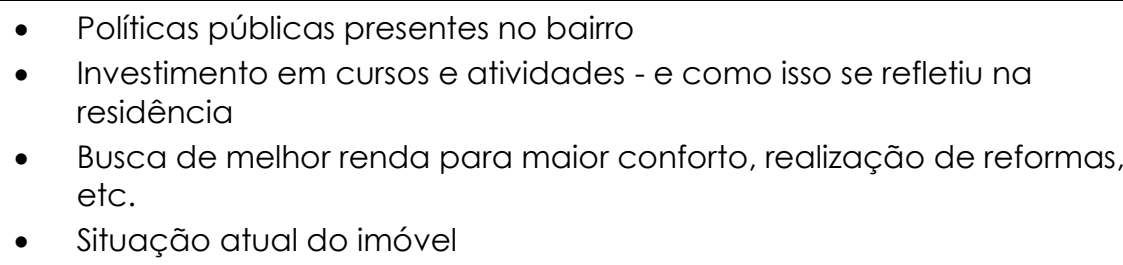 \\
\hline SEGURANÇA & $\begin{array}{l}\text { - } \quad \text { Sensação de insegurança } \\
\text { - } \quad \text { Alterações realizadas na residência, motivadas por insegurança } \\
\text { - } \quad \text { Índice de violência no bairro } \\
\text { - } \quad \text { Força-tarefa de vigilância da vizinhança existente na comunidade }\end{array}$ \\
\hline
\end{tabular}

Fonte: Autoras 2019

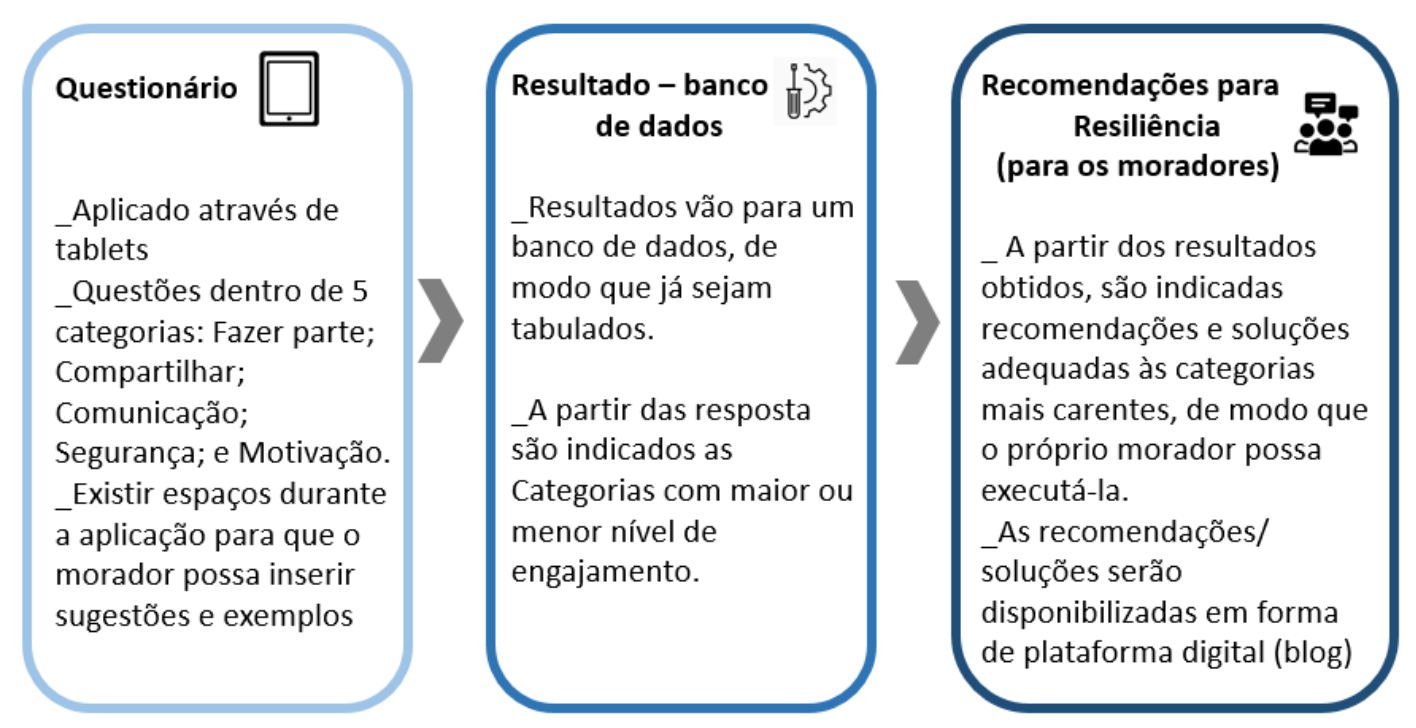

Figura 6 - Funcionamento da ferramenta -

Fonte: Autoras (2019) 


\section{CONSIDERAÇÕES FINAIS}

A identificação e análise dos impactos, a partir de dados previamente levantados, atuam como elementos norteadores da ferramenta de avaliação. A partir dos impactos identificados, juntamente com a futura aplicação, busca-se validar o presente processo investigativo, estabelecendo uma relação positiva entre engajamento e resiliência social no ambiente construído - especificamente em conjuntos de HIS. Com o desenvolvimento e futura aplicação da ferramenta final, busca-se, a partir do uso de plataformas digitais, a divulgação da mesma, de forma que ela possa ser aplicada em diferentes contextos, oferecendo um impacto real, direto e prático, através de soluções direcionadas ao usuário, que funcionem de acordo com as necessidades específicas de cada local. Espera-se que estes resultados fomentem a discussão geral sobre a resiliência como um fator positivo no ambiente construído, dentro de um cenário de vulnerabilidade social.

\section{AGRADECIMENTOS}

À CAPES, pelo apoio recebido.

\section{REFERÊNCIAS}

ADGER, W. N. Social and ecological resilience: are they related? Progress in Human Geography, 24: 347-64. 2000.

ANDERSON, J.; RUGGERI, K.; STEEMERS, K.; HUPPERT, F. Lively Social Space, WellBeing Activity, and Urban Design: Findings From a Low-Cost Community-Led Public Space Intervention. Environment and Behavior. 49, 2016. doi: $10.1177 / 0013916516659108$.

BEAUD, S.; WEBER, F. Guia para a pesquisa de campo: produzir e analisar dados etnográficos. Petrópolis: Vozes, 2007.

BOHM, T. Minha Casa Minha Vida não reduziu déficit habitacional, afirma estudo. Brasília: Jornal do Senado, 2018. Disponível em:

<https://www2.senado.leg.br/bdsf/bitstream/handle/id/538499/Cidadania_622 .pdf? sequence=1>. Acesso em: 27 fev. 2019.

DRESCH, A.; LACERDA, D. P.; ANTUNES JR., J. A. V. DESIGN SCIENCE RESEARCH: Método de Pesquisa para Avanço da Ciência e Tecnologia. Bookman: Porto Alegre, 2017. ISBN 978-85-8260-298-0.

LEAMAN, A.; STEVENSON. F.; BORDASS, B. Building Evaluation: Practice and Principles. Building Research and Information, 38 (5): 564-577, 2010.

LOPES, D. L. et al. O diário de campo e a memória do pesquisador. In: WHITACKER, D. C. A. Sociologia rural: questões metodológicas emergentes. Presidente Wenceslau: Letras à Margem, 2002. p.131-134.

GARCIA, J. E.; VALE, B. Unravelling Sustainability and Resilience in the Built Environment. Routledge, Londres, 2017.

MAGUIRE, B.; CARTWRIGHT, S. Assessing a community's capacity to manage change: A resilience approach to social assessment. Canberra: Australian Government Bureau of Rural Sciences. 2008. 
MALLORY-HILL, S.; PREISER, W. F. E.; WATSON, C. (edits). Enhancing Building Performance. Oxford, UK: Wiley-Blackwell, 2012. 330p.

MARICATO, E. Habitação Social em Áreas Centrais. Revista de Arquitetura e Urbanismo Óculum Ensaios, Faculdade de Arquitetura e Urbanismo - Pontifícia Universidade Católica de Campinas, Campinas, 2000.

MEEROW, S.; NEWELL, J. P. Urban resilience for whom, what, when, where, and why?, Urban Geography, 2016. doi: 10.1080/02723638.2016.1206395

MEEROW, S., NEWELL, J. P., STULTS, M. Defining urban resilience: A review, Landscape and Urban Planning, v. 147, p.38-49, 2015. ISSN 0169-2046, https://doi.org/10.1016/j.landurbplan.2015.11.011.

ONO, R.; ORNSTEIN, S.; VILLA, S.; FRANCA, A. J. G. L. (Org.) Avaliação PósOcupação (APO) na Arquitetura, no Urbanismo e no Design: da Teoria a Prática. 2018.

PETCOU, C.; PETRESCU, D. R-URBAN or how to produce a resilient city. EPHEMERA Theory \& Polytics Organization. 2015. 15 (1). 249 - 262.

PETRESCU, D. M.; PETCOU, C.; BAIBARAC, C. Co-producing commons-based resilience: lessons from R-Urban, Building Research \& Information, 44:7, 717-736, 2016. DOI: 10.1080/09613218.2016.1214891

SCHAUFELI, W.; BAKKER, A. Utrecht work engagement scale: Preliminary manual. Utrecht: Occupational Health Psychology Unit, Utrecht University. 2003.

STEVENSON, F.; PETRESCU, D. Co-producing neighbourhood resilience, Building Research \& Information, 44:7, 695-702, 2016. DOI:

10.1080/09613218.2016.1213865

TRAMONTANO, M. Novos Modos de vida, novos espaços de morar. São Carlos: EES-USP, 1993.

VILLA, S. B. et al. Método de análise da resiliência e adaptabilidade em conjuntos habitacionais sociais através da avaliação pós-ocupação e coprodução. RELATÓRIO FINAL DE PESQUISA: Uberlândia: Universidade Federal de Uberlândia; Universidade de Sheffield, 2017.

VILLA, S. B.; VASCONCELLOS, P. B. COMO VIABILIZAR UNIDADES HABITACIONAIS DE BAIXO CUSTO SOB A ÓTICA DA FLEXIBILIDADE PARA O PROGRAMA MINHA CASA MINHA VIDA? O CASO DO PROJETO MORA [2]. In: $3^{\circ}$ Colóquio de Pesquisa em Arquitetura e Urbanismo e Design Brasil-Portugal: UFU e UTL, 2015, Lisboa. Anais do $3^{\circ}$ Colóquio de Pesquisa em Arquitetura e Urbanismo e Design Brasil-Portugal: UFU e UTL, 2015. v. 1. p. 303-313. 2015.

VILLA, S. B.; SARAMAGO, R. C. P.; GARCIA, L. C. Avaliação Pós-Ocupação no Programa Minha Casa Minha Vida: uma experiência metodológica. 1. ed. Uberlândia: Universidade Federal de Uberlândia, 2015.

VILLA, S. B.; OLIVEIRA, J. C. C. B.; SARAMAGO, R. Respostas ao problema habitacional brasileiro. O caso do projeto MORA. In: $2^{\circ}$ Congresso Internacional da Habitação no Espaço Lusófono ( $\left.2^{\circ} \mathrm{ClHEL}\right), 2013$, Lisboa. Anais do $2^{\circ}$ Congresso Internacional da Habitação no Espaço Lusófono: Habitação, Cidade, Território e Desenvolvimento ( $\left.2^{\circ} \mathrm{CIHEL}\right)$. Lisboa: Laboratório Nacional de Engenharia Civil (LNEC), 2013. v. 1. p. 186-187. 
VILLA, S. B.; ORNSTEIN, S. W. (Org.) Qualidade ambiental na habitação:

avaliação pós-ocupação. São Paulo: Oficina de Textos, 2013. p.359-378.

VILLA, S. B. A APO como elemento norteador de práticas de projeto de HIS. O caso do projeto [MORA]. In: CIHEL 2010. Anais do $1 .{ }^{\circ}$ CONGRESSO

INTERNACIONAL HABITAÇÃO NO ESPAÇO LUSÓFONO, Lisboa - Portugal. 2010. 